\title{
Liquid vortexes and flows induced by femtosecond laser ablation in liquid governing formation of circular and crisscross LIPSS
}

\author{
Dongshi Zhang ${ }^{1,2 \dagger}$, Xinzhuo $\mathrm{Li}^{3 \dagger}$, Yao Fu${ }^{3}$, Qinghe $\mathrm{Yao}^{3 *}$, Zhuguo $\mathrm{Li}^{1,4 *}$ \\ and Koji Sugioka ${ }^{2 *}$
}

\begin{abstract}
Orientations of laser induced periodic surface structures (LIPSS) are usually considered to be governed by the laser polarization state. In this work, we unveil that fluid dynamics induced by femtosecond (fs) laser ablation in liquid (fs-LAL) can easily break this polarization restriction to produce irregular circular-LIPSS (CLIPPS) and crisscross-LIPSS (CCLIPSS). Fs laser ablation of silicon in water shows formation of diverse LIPSS depending on ablation conditions. At a high power of $700 \mathrm{~mW}$ (repetition rate of $100 \mathrm{kHz}$, pulse duration of $457 \mathrm{fs}$ and wavelength of $1045 \mathrm{~nm}$ ), single/twin CLIPSS are produced at the bottom of macropores of several microns in diameter due to the formation of strong liquid vortexes and occurrence of the vortex shedding effect. Theoretical simulations validate our speculation about the formation of liquid vortex with an ultrahigh static pressure, which can induce the microstructure trenches and cracks at the sidewalls for fs-LAL of Si and tungsten (W) in water, respectively. At a low power of $50 \mathrm{~mW}$, weak liquid vortexes are produced, which only give birth to curved LIPSS in the valleys of grooves. Consequently, it is deduced that liquid vortex plays a crucial role in the formation of macropores. Mountain-like microstructures induce complex fluid dynamics which can cause the formation of CCLIPSS on them. It is believed that liquid vortexes and fluid dynamics presented in this work open up new possibilities to diversify the morphologies of LIPSS formed by fs-LAL.
\end{abstract}

Keywords: circular LIPSS; crisscross LIPSS; laser ablation in liquid; femtosecond laser ablation in water; liquid vortex; vortex shedding

Zhang DS, Li XZ, Fu Y, Yao QH, Li ZG et al. Liquid vortexes and flows induced by femtosecond laser ablation in liquid governing formation of circular and crisscross LIPSS. Opto-Electron Adv 5, 210066 (2022).

\section{Introduction}

Ultrashort (femtosecond (fs) and picosecond (ps)) pulse laser ablation/processing (UPLA/P) is occupying an increasingly important position in the manufacturing field because of some specific merits ${ }^{1,2}$. In particular, UPLA/P can produce extremely high pressure, high temperature
(HPHT) environment ${ }^{3-5}$ in atmosphere, which is not available by conventional lasers such as continuous wave and nanosecond lasers. This feature offers versatility of UPLA/P to realize surface structuring, nanomaterial synthesis and nanomaterial assembly ${ }^{6}$. Adjusting UPLA/P environment and changing the laser/processing

\footnotetext{
1Shanghai Key Laboratory of Materials Laser Processing and Modification, School of Materials Science and Engineering, Shanghai Jiao Tong University, Shanghai 200240, China; ${ }^{2}$ RIKEN Center for Advanced Photonics, 2-1 Hirosawa, Wako, Saitama 351-0198, Japan; ${ }^{3}$ School of Aeronautics and Astronautics, Sun Yat-sen University, Guangzhou 510275, China; ${ }^{4}$ State Key Lab of Metal Matrix Composites, School of Materials Science and Engineering, Shanghai Jiao Tong University, Shanghai 200240, China.

tThese authors contributed equally to this work.

"Correspondence: QH Yao, E-mail: yaoqhe@mail.sysu.edu.cn; ZG Li, E-mail: lizg@sjtu.edu.cn; K Sugioka, E-mail: ksugioka@riken.jp Received: 20 May 2021; Accepted: 13 August 2021; Published online: 28 February 2022
}

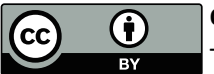

Open Access This article is licensed under a Creative Commons Attribution 4.0 International License.

To view a copy of this license, visit http://creativecommons.org/licenses/by/4.0/.

(C) The Author(s) 2022. Published by Institute of Optics and Electronics, Chinese Academy of Sciences. 
parameters (e.g., pulse energy, pulse duration, repetition rate, scan speed, etc. $\left.{ }^{3,7}\right)$, varying experimental setups ${ }^{8}$ and target materials ${ }^{9-10}$ render high flexibility to this technique, enabling synthesis of different types of nanomaterials and formation of diverse structures. As far as the surface structuring is concerned, the created structures can involve the nanoscale, microscale or multiscale, which make them applicable for different applications in the fields of catalysis, biology, optics and optoelectronics, since the structure scale highly influences surface properties such as wettabilty, adhesiveness, sensitivity, color and absorption ${ }^{11-16}$. Additionally, UPLA/P is capable of surface structuring of a large variety of materials including metals, ceramics, semiconductors, dielectrics, polymers, and two-dimensional materials ${ }^{17-27}$, which involve hard-to-treat materials ${ }^{26}$. Recent UPLA/P innovations have unveiled that new factors such as electric and magnetic field ${ }^{28,29}$, high-pressure shockwave ${ }^{30}$, and persistent bubbles ${ }^{18,31}$ generated during UPLA/P or exerted externally may become new keys to manipulate structure morphologies. Despite the success in generating new types of structures at different scales, it is still challenging to further enrich the structural diversity and unveil the underlying key factors that cause the differences.

Laser induced periodic surface structures (LIPSS) ${ }^{32,33}$ is an evergreen subject since its discovery in $1965^{34}$ owing to its ease to be constructed on different materials by single-step laser irradiation/ablation for optical, eletrochemical, biological and mechanical application $s^{32,35}$. It is commonly found that low spatial frequency LIPSS (LSFL) on high-absorbance materials (metals) and low-absorbance materials (dielectrics) are oriented perpendicular and parallel to the polarization direction of the linearly polarized laser utilized ${ }^{16}$, respectively. Controlling the polarization directions of linearly polarized lasers allows flexibly manipulating the orientations of LIPSS ${ }^{36-38}$ to generate different colors on the surfaces, which opens up new opportunities for colorful paintings of metallic surfaces by programming the orientations of LIPSS ${ }^{39,40}$. In comparison, circularly polarized laser beams often give rise to the formation of bead-strings ${ }^{41}$. Thanks to the advance in technique methodology and optics, non-linear LIPSS have already become accessible. For example, Han et al. constructed concentric semi circular LIPSS (CLIPSS) on silicon by single-pulse fs laser irradiation on the pre-processed quasi-plasmonic annular-shaped nanostructures ${ }^{42}$, where the excited surface plasmon polaritons (SPPs) triggered by laser irradiation played a key role in its formation. Romashevskiy et al. produced
CLIPSS on silicon via bubble-diffracted Gaussian-beam fs laser pulses ${ }^{43}$. A series of radial- and ring-shaped LIPSS have been created by laser ablation using radially and azimuthally polarized vortex beams, respectively ${ }^{44-47}$, in which the orientations of nonlinear LIPSS are still perpendicular to the directions of local beam polarizations $s^{45}$. In our previous work, we showed that the orientations of high spatial frequency LIPSS (HSFL) were not strictly perpendicular to the direction of light polarization ${ }^{48}$, and possess a maximal tilt angle of $50^{\circ}$ on the microstructures with height gradients. Both clock-wise and anticlockwise tilted HSFL were found, which converged together in a sink-like inlet and resulted in the formation of normal HSFL in the outlet ${ }^{48}$. These findings indicate that an influential factor must be triggered during fs laser ablation in liquid (fs-LAL) ${ }^{3}$, which can compromise the effect of light polarization on the orientations of LIPSS. However, to date, there is no evidence to confirm what this factor is and whether it is possible to induce CLIPSS using a linearly polarized laser without any external assistance. Tsibidis et al. proposed a hybrid model to interpret the structural evolution from LIPSS to microspikes and concluded that surface melt in combination with electromagnetic interference led to the formation of LIPSS, while hydrodynamics including Marangoni convection and counter- rotating vortex rolls led to the formation of microgrooves and microspikes ${ }^{49}$. Hence, it is deduced that liquid vortex may be generated during laser ablation in liquids, which may give birth to CLIPSS structures.

In this paper, we demonstrate the possibility to induce formation of CLIPSS or crisscross LIPSS (CCLIPSS) by linearly polarized fs laser ablation of silicon in water depending on the conditions. Macropores with a dimension in the microscale are speculated to be the ideal candidates to identify the influential factor responsible for LIPSS tilting and CLIPSS production because of the height gradients in all directions. Hence, a special attention is paid to the nanostructures in macropores formed in the valley of the grooves. Fs-LAL of tungsten (W) in water is further carried out to check how ultrahigh pressures of LAL-induced liquid vortexes influence the side walls of the microstructures. Three models including single macropore, four macropore arrays, and mountainlike bumps are established for simulation verification.

\section{Experimental}

An fs laser system (FCPA $\mu$ Jewel D-1000-UG3, IMRA America Inc., Ann Arbor, MI, USA) with a pulse 
duration, wavelength and repetition rate of, respectively, $457 \mathrm{fs}, 1045 \mathrm{~nm}$ and $100 \mathrm{kHz}$ was used for fs-LAL. A $20 \times$ objective lens (numerical aperture (NA): 0.4, Mitutoyo, Kawasaki, Japan) was used to focus the laser beam on a single-crystalline silicon substrate placed inside a glass culture dish (diameter of $45 \mathrm{~mm}$, height of $20 \mathrm{~mm}$ ) filled with $8 \mathrm{~mL}$ water. The liquid thickness above the target surface was kept constant to be $5 \mathrm{~mm}$. Two different laser powers of 700 and $50 \mathrm{~mW}$ were employed for fs$\mathrm{LAL}$ of $\mathrm{Si}$, while a fixed laser power of $600 \mathrm{~mW}$ was used for fs-LAL of W. The pulse energies for 700, 600 and 50 $\mathrm{mW}$ correspond to 7, 6 and $0.5 \mu \mathrm{J}$, respectively. The laser spot size was estimated to be $3.4 \mu \mathrm{m}$ based on $\lambda=1045$ $\mathrm{nm}$ and $M^{2}=1.1$ of our laser system and $N A=0.4$ of the objective lens used, so the fluences for laser powers of 700,600 , and $50 \mathrm{~mW}$ at $100 \mathrm{kHz}$ repetition rate were calculated to be around $77.1,66.1$ and $5.5 \mathrm{~J} / \mathrm{cm}^{2}$, respectively. An area of $2 \mathrm{~mm} \times 2 \mathrm{~mm}$ was scanned for each case using the line-by-line scanning described in ref. ${ }^{50}$. The scanning line intervals were 15 and $5 \mu \mathrm{m}$ for 700 $\mathrm{mW}$-fs-LAL of Si, $5 \mu \mathrm{m}$ for $50 \mathrm{~mW}$-fs-LAL of $\mathrm{Si}$ and 600 $\mathrm{mW}$-fs-LAL of W. A scanning electron microscope (SEM) (Thermo Fisher Scientific, Quattro ESEM, Tokyo, Japan) was used to characterize surface structure morphologies including both microstructures and HSFL with different orientations.

Selecting these experimental parameters is due to the following reasons. During the characterization of the structures generated by ablation at a laser energy of 700 $\mathrm{mW}$ with different scan intervals of 5 and $15 \mu \mathrm{m}^{30}$, CLIPSS and CCLIPSS structures were discovered, which are the main content of this work. To prevent a repetitive description of the same results, this manuscript only displays the results about CLIPSS and CCLIPSS. To demonstrate the reproducibility of the experiments, we performed laser ablation of $\mathrm{Si}$ in water at the laser powers of $50 \mathrm{~mW}$ to see how structures evolve when weaker liquid flows are generated. Regarding the cracking phenomenon induced by liquid vortexes during ablation of $\mathrm{W}$, we have already reported the morphology of $600 \mathrm{~mW}$-laser ablated $\mathrm{W}^{51}$ and $\mathrm{Si}^{48}$. Our previous works reported the interesting discovery of abnormally oriented LIPSS on height-gradient microstructures ${ }^{48}$, but the mechanism for this abnormal phenomenon was only speculated based on previous LIPSS formation mechanisms. This work is well supplementary to our previous works because it for the first time reveals the impact of liquid flows on LIPSS formation.

\section{Simulation}

The simulation does not consider the impacts of shockwaves and laser-matter interactions. We only assume the liquid moving velocity is the same as that of the shockwave. The reason for the simple modeling is because of the high complexity of multi-pulse ablation in liquids, including generation and quenching of plasma, shockwave reflection ${ }^{52}$, generation and collapse of bubbles ${ }^{53}$, light reflection/refraction by bubbles ${ }^{18,54}$, surface oxidation and excited surface plasmon polaritons (SPPs) ${ }^{55}$. No matter which complex condition is formed, a high-speed shockwave must be produced by laser ablation and will serve as the driving force for the generation of liquid flows. The lifetime of shockwaves is around $200 \mathrm{~ns}^{56}$. The distances between adjacent grooves are 5 and $15 \mu \mathrm{m}$. The shockwave speed is $\sim 5000 \mathrm{~m} / \mathrm{s}^{57}$. This means that after 1 and $3 \mathrm{~ns}$, the shockwave will be reflected between adjacent grooves, which should be strong enough to induce the high-speed flow among the grooves. The lifetime of molten layers (the precursors of LIPSS) is longer than 30 $\mathrm{ns}$ in the case of laser ablation in $\mathrm{air}^{58}$. In water, benefiting from liquid cooling, the lifetime of molten layers is reduced to several $n s^{59}$. From the perspective of timescale, surface melting, SPPs and shockwave propagation can take place simultaneously. Therefore, the simulation in our study corresponds to the experiment to some extent, with a focus on the influence of fluid flows on different structures in a molten state (precursor of LIPSS $^{48,60}$ ) in the presence of SPPs excited by laser ablation.

Traditional simulation methods are not suitable to realize an ultra-high-speed fluid simulation in the microscale because very rigorous laws in the microscale have to be employed. In the case that the basic assumptions and axioms established by the basic framework of continuum mechanics cannot be fully satisfied, the rationality of amplification method is strongly illustrated. With the same Reynolds number (Re) and Knudsen number $(\mathrm{Kn})$, the internal flow fields of the microscale sample and the amplified milliscale sample have a high similarity. Therefore, in our simulation, for simplicity, we used the amplified milliscale models to simulate the microscale fluid mechanics in the extreme environment of ultra-high-speed water flows. Re is a non-quantitative number that can be used to characterize fluid flow. When Re is small, the influence of the sticky force on the flow field is greater than the inertia, so that the 
disturbance of the flow rate in the flow field will be attenuated by the viscous force. The fluid flow is stable, which is normally a stratospheric flow. Kn represents the ratio of the average free range of molecules to the characteristic length $L$ of an object in the flow field. Kn and Re can be calculated using the following equations

$$
\begin{gathered}
K n=\frac{\lambda}{L}=\frac{4 K_{\mathrm{b}} T}{\sqrt{2} \pi \sigma^{2}} \times \frac{1}{p L}, \\
R e=\frac{\rho u L}{\mu}=\frac{4 m_{\mathrm{r}}}{\pi L \mu},
\end{gathered}
$$

where $\lambda$ is the free path of the molecule, $L$ is the geometric feature scale which is set as the macropore size and groove depth, $K_{\mathrm{b}}$ is the Boltzmann constant, $\sigma$ is the molecular diameter, $p$ is the fluid pressure, i.e. qualitative pressure of $K n$, and $T$ is the fluid thermodynamic temperature. The $K n$ is mainly determined by geometric scale and fluid pressure. The domain outlet fluid pressure was selected as the qualitative pressure of the small macropore fluid $K n$. In Eq. (2), $\mu$ is the kinetic viscosity of a fluid, which is set as a constant for simulation. $\rho$ is fluid density, $u$ is fluid characteristic speed, $L$ is geometric feature, $m_{\mathrm{r}}$ is mass flow. Re can be changed by changing the fluid characteristic speed $u$.

Under the continuous medium condition, when the Reynolds number of the microfluid and its amplified milli-scale fluid are equal, they have proven to possess very similar flow characteristics ${ }^{61}$. Hence, in this work, we performed the simulation of the magnified milli-scale model under the continuous medium hypothesis with the same Knudsen number, to solve the difficulties of grid generation and convergence for micron scale structures. The full size of model was about $10 \mu \mathrm{m}$ according to the sizes of macropores published in our previous report $^{30}$. The parameters for the amplified milli-scale simulation models were set at $L=10 \mathrm{~mm}, u=5 \mathrm{~m} / \mathrm{s}$, and $p=$ $1.01 \times 10^{3} \mathrm{~Pa}$. In reality, the pressure of fluids should vary with time and structures. Shockwave reflection between the grooves will complicate the flow direction and pressures. Considering a pulsed laser with a repetition rate of $100 \mathrm{kHz}$ used in this study, the time interval between two successive pulses is $10 \mu \mathrm{s}$, which is much shorter than the lifetime of cavitation bubbles generated during laser ablation in liquids ${ }^{62}$. This means that subsequent pulses will hit the cavitation bubbles to induce their breakdown, while the bubbles will also reflect/refract the laser beam $^{18,54}$, making the processes very complex. The collapse of cavitation bubbles also generates shockwaves ${ }^{63}$, which may also influence the pressure of liquid flows.
Hence, for simplicity, we assume that the fluid is kept always at a constant pressure for simulation. Since the shockwave is formed after plasma quenching ${ }^{3}$, the temperature of liquid flow induced by the propagation of shockwave is assumed to be room temperature.

The fluid medium was water. The Kn value was calculated to be $\sim 10^{-13}$ according to the geometric and pneumatic parameters. $\mathrm{Kn}$ is generally used to determine whether the fluid is suitable for the continuous assumptions. It is generally believed that when $\mathrm{Kn}$ is smaller than 0.001 , the liquid flow can be categorized as a continuous medium ${ }^{64}$. According to Kn value we calculated, the liquid flow induced by strong shockwaves can be categorized as a continuous medium. Hence, three hypotheses for Navier-Stokes equation are met, including linear relation between stress tension and strain rate tension, isotropic fluid and zero stationary fluid strain rate.

Navier-Stokes equation:

$\rho \frac{D v}{D t}=\rho \boldsymbol{F}_{b}-\operatorname{grad} p+\operatorname{div}(2 \mu \boldsymbol{S})-\frac{2}{3} \operatorname{grad}(\mu \operatorname{div} v)$.

Reynolds average of Navier-Stokes equation, we can get:

$\rho \frac{\partial \overline{u_{i}}}{\partial t}+\rho \overline{u_{j}} \frac{\partial \overline{u_{i}}}{\partial x_{i}}=-\frac{\partial \bar{p}}{\partial x_{i}}+\mu \frac{\partial^{2} \overline{u_{i}}}{\partial x_{i} \partial x_{j}}-\rho \frac{\partial^{2} \overline{u_{i}^{\prime} u_{j}^{\prime}}}{\partial x_{j}}+\rho \overline{f_{i}}$

where $\rho$ is liquid density, $p$ is hydrostatic pressure, $\overline{u_{i}}$ is speed component, $\bar{f}_{i}$ is mass force, $\mu$ is dynamic viscosity coefficient, $\overline{u_{i} u_{j}^{\prime}}$ is Reynolds stress. Because of the presence of Reynolds stress, this equation is not closed. To make the equation closed and for the sake of computational efficiency, the following RNG K- $\varepsilon$ turbulent model ( $\mathrm{k}$ equation) is adopted.

Turbulent kinetic energy equation of $\mathrm{K}-\varepsilon$ turbulent model is:

$\frac{\partial(\rho k)}{\partial t}+\frac{\partial\left(\rho k \bar{u}_{i}\right)}{\partial x_{i}}=\frac{\partial}{\partial x_{j}}\left[\left(\mu+\frac{\mu_{t}}{\sigma_{k}}\right) \frac{\partial k}{\partial x_{j}}\right]+G_{k}-\rho \varepsilon$.

Turbulent dissipation rate equation ( $\varepsilon$ equation) is:

$$
\begin{aligned}
& \frac{\partial(\rho \varepsilon)}{\partial t}+\frac{\partial\left(\rho k \varepsilon \bar{u}_{i}\right)}{\partial x_{i}}=\frac{\partial}{\partial x_{i}}\left[\left(\mu+\frac{\mu_{t}}{\sigma_{k}}\right) \frac{\partial \varepsilon}{\partial x_{j}}\right]+C_{\varepsilon 1} \frac{\varepsilon}{k} G_{k} \\
& -\left(C_{\varepsilon 2}+\frac{C_{\mu \eta^{3}}\left(1-\eta / \eta_{0}\right)}{1+\beta \eta^{3}}\right) \rho \frac{\varepsilon^{2}}{k}
\end{aligned}
$$

where $\mu_{t}=\rho C_{\mu} \frac{k^{2}}{\varepsilon}$ is turbulent viscosity term, $G_{k}=\mu_{t} S^{2}, \quad S=\sqrt{2 S_{i j} S_{i j}}, \quad S_{i j}=\frac{1}{2}\left(\frac{\partial u_{i}}{\partial x_{j}}+\frac{\partial u_{j}}{\partial x_{j}}\right), \quad \eta=\frac{S k}{\varepsilon}$ are turbulent kinetic energy production terms. 
Considering the flow on the microscale structures had a strong pressure drop and compressibility and the viscosity force changed significantly with increasing the flow surface area to volume ratio, the enhanced wall function was selected. Wall surfaces were set as the nonslip boundary condition for simulation. We used the Leap Squares Cells Based method to make the gradient item in equation (3) discrete, and adopt the second-order precision upwind format to make N-S equation and $\mathrm{K}-\varepsilon$ turbulent model discrete. The tolerance of convergence criteria is $10^{-4}$ for our simulation.

Laser ablation in liquid normally induces the formation of stress waves in the ablated solid target and shockwaves in liquids ${ }^{65}$, which are the driving forces for the breaking of brittle structures. The propagation of shockwaves may push the liquid to flow horizontally on the surfaces, which is deemed to be the dominant flow driving force. The water flow is driven by the laser induced shockwaves at a speed of $\sim 5000 \mathrm{~m} / \mathrm{s}^{57}$, which is assumed to be the same as the speed of liquid flows generated by fs-LAL in water for simulation. During laser ablation, water breakdown also takes place in the beam path, leading to the formation of many nanobubbles ${ }^{66}$. The collapse of these nanobubbles induces the formation of another series of shockwaves and results in the formation of vertical liquids flowing towards the ablated surfaces. When small bubbles are subjected to laser irradiation, very complex Marangoni flows can be induced ${ }^{67}$, which leads to the generation of complex tilt flows along different directions. Hence, superimposed fluid flows coming from both horizontal and vertical directions were adopted for simulations of the flow fields on the structures. The simulation model is a little enlarged for the mountain-like structure to demonstrate the possibility of inducing crisscross flows, which is aimed to ensure different directional flows on different parts of the structures to be seen more clearly. The height and radius of the simulated mountain-like structure are 6 and $10 \mu \mathrm{m}$, respectively. A small trench with a depth of $2 \mu \mathrm{m}$ is assumed to be located beneath the mountain-like structure. The starting flow is horizontal from upper right to bottom left at a speed of $5 \mathrm{~km} / \mathrm{s}$.

\section{Results and discussion}

\section{CLIPSS}

Figure 1(a,b) show the side and top views of hierarchical micro/nanostructures created by fs-LAL of Si in water at a laser power of $700 \mathrm{~mW}$, a scan interval of $15 \mu \mathrm{m}$ and a scan speed of $1 \mathrm{~mm} / \mathrm{s}$. Most grooves are broken due to the plasma confinement in them and the peening effect of strong shockwaves with the pressure amplitude of $\mathrm{GPa}^{3}$. The broken grooves are characterized by sharp edges (Fig. 1(b)), very different from the unbroken grooves ${ }^{48}$. The smoothly broken regions show a dark color, while the other regions displaying a white color are decorated by HSFL with periods of 100-200 nm (Inset image in Fig. 1(b)), which are formed due to the generation of surface plasmon polaritons ${ }^{68,69}$. The orientation of HSFL is perpendicular to that of the light polarization, in accordance with previous reports ${ }^{32,70,71}$. Inhomogeneous macropores are generated in the valleys of broken grooves (Fig. 1(b)), which indicates that there is no need of multiple back-and-forth runs of fs laser ablation to generate macropores since the line-by-line scanning have been employed in this study ${ }^{72}$. CLIPSS is located in the deep macropores, as shown in Fig. 1(c-f), including both single CLIPSS (Fig. 1(c, d)) and twin CLIPSS (Fig. 1(e, f)). It is noteworthy that according to our observation, the formation of single CLIPSS and twin CLIPSS are randomly generated because of the complexity of the multipulse ablation conditions.

To check the dependence of CLIPSS formation on the laser parameters, LAL at a lower power of $50 \mathrm{~mW}$ was performed with the results shown in Fig. 1(g-l). Figure $1(\mathrm{~g})$ and $1(\mathrm{~h})$ display the morphologies of shallow grooves obtained by $50 \mathrm{~mW}$-fs-LAL. To clearly witness the macropores, a high contrast image was taken as shown in Fig. 1(g). Due to the lower laser fluence employed, the grooves are intact without any breaking. The as-prepared grooves are in a wavy manner (Fig. 1(g, h)), not straight to the scanned lines ${ }^{48}$, which is attributed to the formation of macropores inside the valleys of grooves (Fig. 1(g)). Figure 1(i) and 1(k) show the enlarged morphologies of the green and blue open rectangle regions in Fig. 1(g), respectively, where incomplete CLIPSS are found at the bottom of macropores. Curved layered structures with one nanoprotrusion in the central region are found in Fig. 1(i). Depicting the curved layered structures by a green dash line clearly exhibits that the structures are actually in a spiral shape like a liquid vortex. Figure $1(\mathrm{k})$ shows the possibility to produce irregular LIPSS in one macropore, whose orientation is not perpendicular to the light polarization direction. This finding indicates that another directional force, highly brought by the liquid vortex, causes the deviation of the LIPSS' orientations. To check the influential area of liquid vortexes, we examined the orientations of HSFL on 


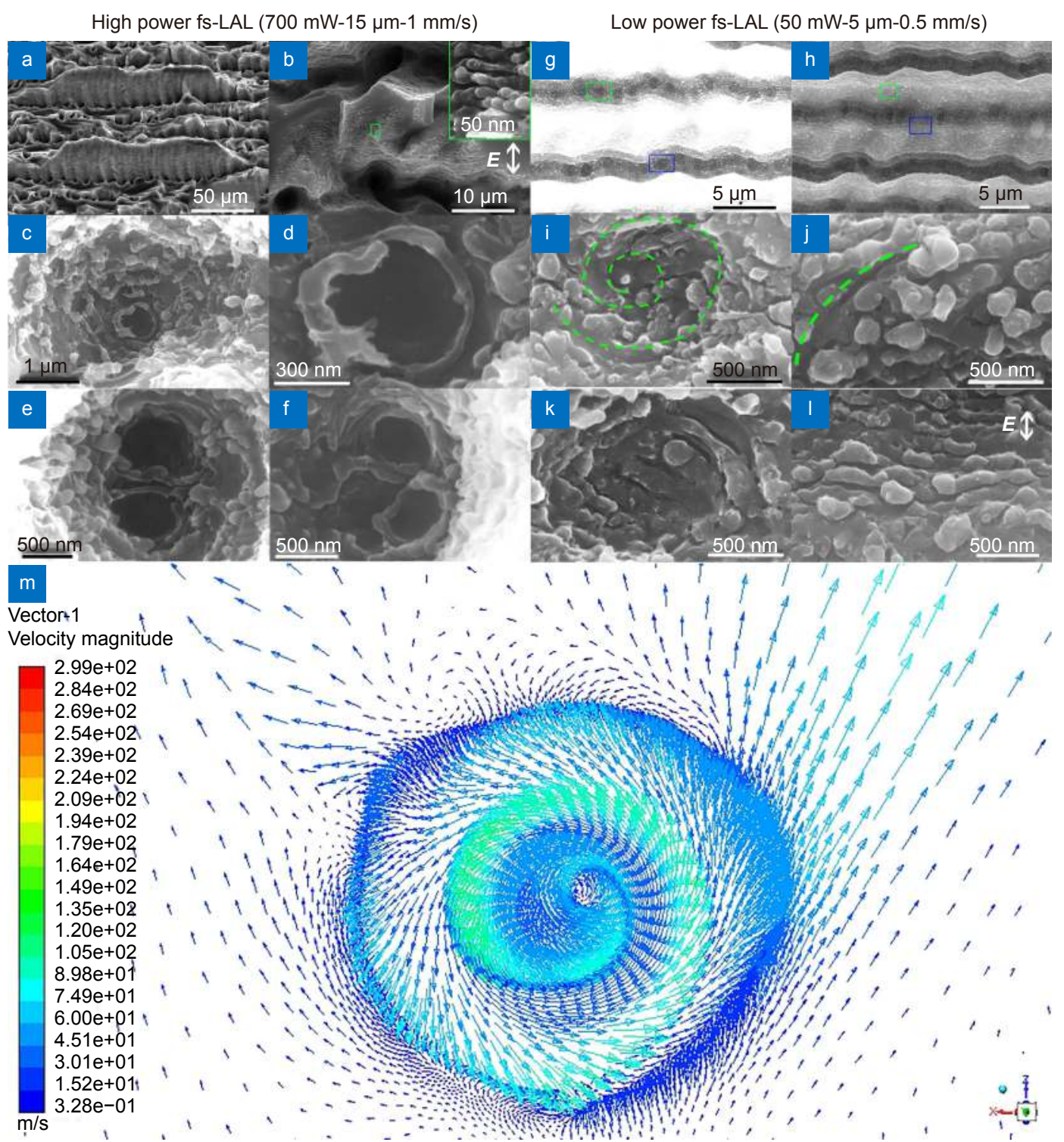

Fig. 1 | (a-b) SEM images showing the side and top views of the cracked structures obtained by fs-LAL of Si at a laser power of $700 \mathrm{~mW}$, a scan interval of $15 \mu \mathrm{m}$, and a scan speed of $1 \mathrm{~mm} / \mathrm{s}$. (c) Structure of a macropore. (d) Enlarged CLIPSS morphology in (c). (e, f) Twin CLIPSS in a macropore. (g, h) SEM images of the grooves obtained by 50mW-fs-LAL. High contrast applied to $(\mathrm{g})$ is to see macropores in the valleys of grooves more clearly. (i/k, $\mathbf{j} / \mathbf{l})$ Enlarged images of green and blue rectangles in $(\mathrm{g})$ and $(\mathrm{h})$, respectively. The direction of laser polarization is shown in (b, I). (i, j, k) and (I) Irregular and normal LIPSS, respectively. (m) Velocity vector of a fluid vortex formed in a hole structure upon the impact of high-speed superimposed horizontal and vertical flows of fluids.

the crests of grooves (green and blue open rectangle regions in Fig. 1(h)). The HSFL on the crest is curved (Fig. $1(\mathrm{j}))$, as indicated by the green dash lines. This means that a liquid vortex is large enough to affect the orientations of LIPSS on the crests of macropores, which is the reason why the grooves become wavy-like (Fig. 1(g, h)). Only in the joint area of macropores (Fig. 1(1)), normal LIPSS whose orientation is perpendicular to the light polarization direction are found (Fig. 1(1)). Figure. 1(c-f) and $1(\mathrm{i}-\mathrm{k})$ give strong evidences to confirm the formation of liquid vortexes during fs-LAL.
To validate our speculation, we simulate the scenario when a macropore is subjected to the superimposed liquid flows coming from both vertical and horizontal directions. Figure $1(\mathrm{~m})$ proves the possibility to induce a liquid vortex in a macropore. The simulated results show that the liquid vortex has a velocity distribution of $200-700 \mathrm{~m} / \mathrm{s}$, which obviously decelerates in the macropore. Figure 1(i) indicates that one liquid vortex is generated in each macropore. Considering many macropores are found in the broken grooves, it is deduced that many liquid vortexes are formed during fs-LAL. Analogously, 
the formation of periodic macropores by laser ablation in air is also attributed to the formation of gas vortexes in valleys of grooves ${ }^{72-75}$. When two liquid vortexes are generated in one macropore, the vortex shedding effect takes place $^{76}$, which causes the formations of twin CLIPSS.

Figure 2(a,b) display the side and top views of pressure nephograms of the simulated liquid vortex. Except for the central point of the liquid vortex (Fig. 2(b)), the stress gradually increases as the depth increases (Fig. 2(a)) with a maximal pressure of $8.1 \times 10^{5} \mathrm{kPa}$. Since the liquid vortex can exert an ultra-high pressure to the side walls of the formed structures, it may induce severe cracks to a brittle material such as tungsten (W). Previous reports have shown that metal $\mathrm{W}$ can be easily cracked upon the impact of laser pulses when the pressure exceeds its tensile stress limit ${ }^{77,78}$. To investigate whether the liquid vortexes can cause cracks to the microstructures, we checked the ablated $\mathrm{W}$ sample we have prepared for antireflective studies ${ }^{51}$. This sample was prepared by fs-LAL of $\mathrm{W}$ in water at a laser power of 600 $\mathrm{mW}$, a scan interval of $5 \mu \mathrm{m}$, and a scan speed of $1 \mathrm{~mm} / \mathrm{s}$. In accordance with our speculation, liquid vortexes gen- erated by fs-LAL in water are capable of inducing many horizontal cracks on the side walls of ablated $\mathrm{W}$ grooves (Fig. 2(c)). The cracks propagate through the whole side walls. This means that the liquid vortexes are movable during laser ablation or intense liquid vortexes which can influence all ablated regions generated during fs-LAL in water. Figure $2(\mathrm{~d}, \mathrm{e})$ and $2(\mathrm{f})$ display the morphologies of the side walls of the grooves generated by fs-LAL of $\mathrm{Si}$ in water at a fixed condition of a laser power of 700 $\mathrm{mW}$ and a scan interval of $15 \mu \mathrm{m}$ with two different scan speeds of 0.5 and $1 \mathrm{~mm} / \mathrm{s}$, respectively. The side walls of grooves are characterized by curved trenches whose bending directions change with the scan directions (Fig. $2(d, e)$ ). Hence, it can be concluded that the liquid vortexes are indeed movable as the scan direction changes. At the fixed laser power, the density of liquid vortexes varies with the scan speed. The lower the scan speed $(0.5$ vs $1 \mathrm{~mm} / \mathrm{s}$ ), the higher intensity of the liquid vortexes, as indicated by the decreased curved trench distance from 5 (Fig. 1(d, e)) to $10 \mu \mathrm{m}$ (Fig. 1(f)). Tuerke et al. simulated the flow fields of a double-cavity trench to demonstrate the possibility of simultaneously inducing two liquid

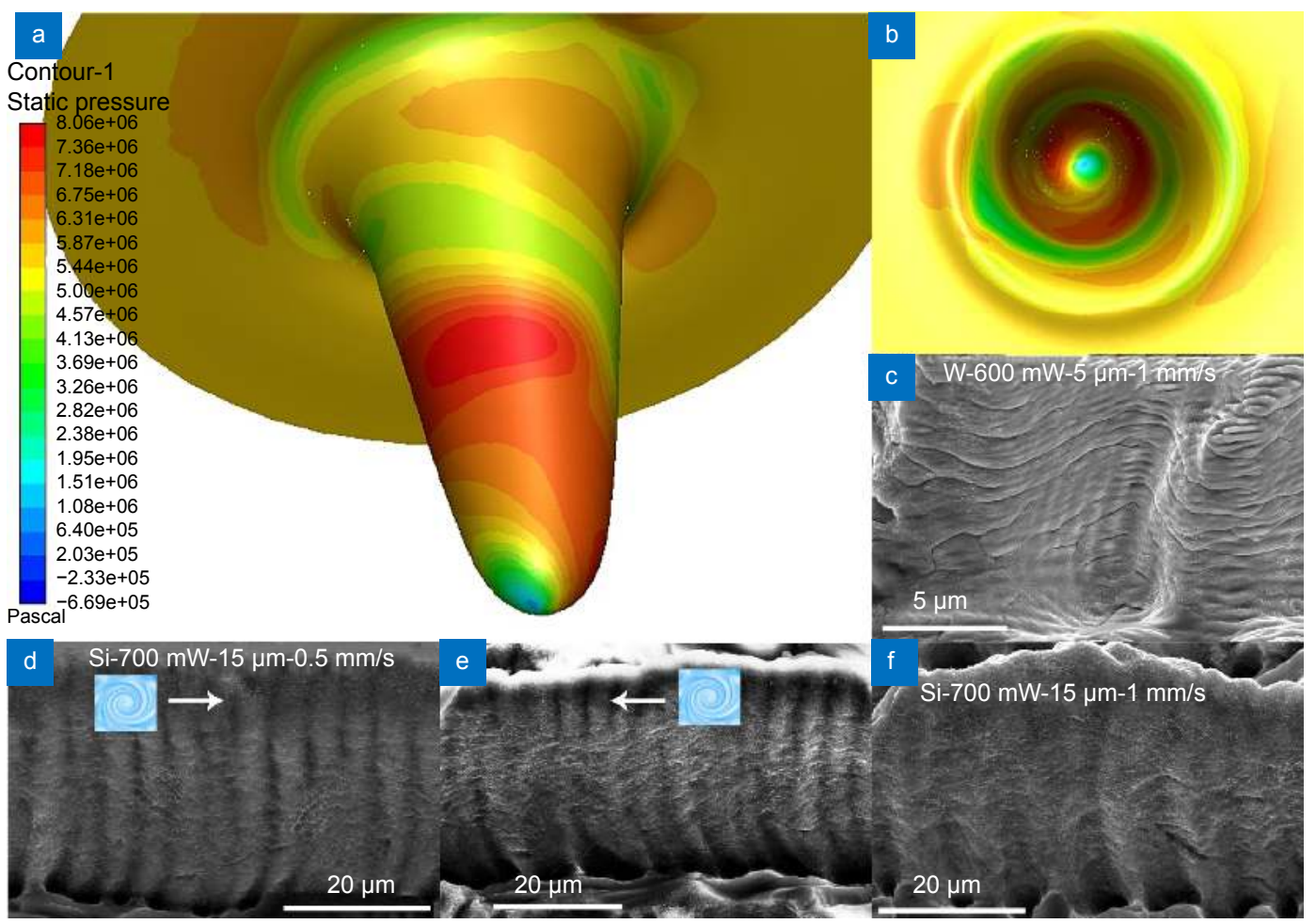

Fig. 2 | (a) Side view and (b) top view of the static pressure distribution of the simulated liquid vortex. (c) SEM image of the tungsten groove created by fs-LAL of $W$ in water at a laser power of $600 \mathrm{~mW}$, a scan interval of $5 \mu \mathrm{m}$, and a scan speed of $1 \mathrm{~mm} / \mathrm{s}$, which indicates that liquid vortexes induce a series of horizontal cracks. (d, e) and (f) SEM images showing the morphologies of the side walls of grooves generated by fs-LAL of $\mathrm{Si}$ in water at fixed conditions of a laser power of $700 \mathrm{~mW}$ and a scan interval of $15 \mu \mathrm{m}$ with two different scan speeds of $0.5 \mathrm{and} 1 \mathrm{~mm} / \mathrm{s}$, respectively. SEM images were taken from the side view with a $30^{\circ}$ tilt. 
vortexes in two cavities ${ }^{79}$. In our case, the facing trenches on the sidewalls of adjacent grooves (Fig. 2(d, e)) can play the same role as two cavities. Hence, upon the impact of a strong liquid flow brought by the successive laser ablation, twin liquid vortexes can be triggered in the macropore trenches, which can cause the formation of twin CLIPSS inside one macropore.

Simulation and experimental results shown in Fig. $3(\mathrm{a}-\mathrm{c})$ also indicate that when a string of many macropores are formed, the liquid vortexes can be simultaneously generated on the side walls of grooves. Five layered CLIPSS can be identified from the left crater shown in Fig. 3(c). One irregular elliptical CLIPSS is produced on the right side, whose irregularity may be caused by the mobility of the formed liquid vortex. Since the pressure of liquid vortex in the deepest part is not so strong (Fig. 2(a, b)), the central regions of CLIPSS are re- latively flat (Fig. 3(c)).

\section{CCLIPSS on mountain-like microstructures}

Microstructural geometry has been proven to be a crucial factor to determine the flow field ${ }^{80,81}$. Due to the occurrence of a series of cracks in the grooves, very complex corrugated microstructures must have been generated during fs-LAL as shown in Fig. 4(a), which can significantly influence the fluid motion states. Figure 4(b) displays the enlarged mountain-like structure of the green rectangle region in Fig. 4(a), where two orientational LIPSS are found to converge at the bottom of the mountain-like microstructures, as indicated by two green arrows. The orientations of LIPSS are no longer perpendicular to the direction of light polarization but change according to the microstructural curvatures, in accordance with our previous finding ${ }^{48}$. Figure $4(\mathrm{c})$
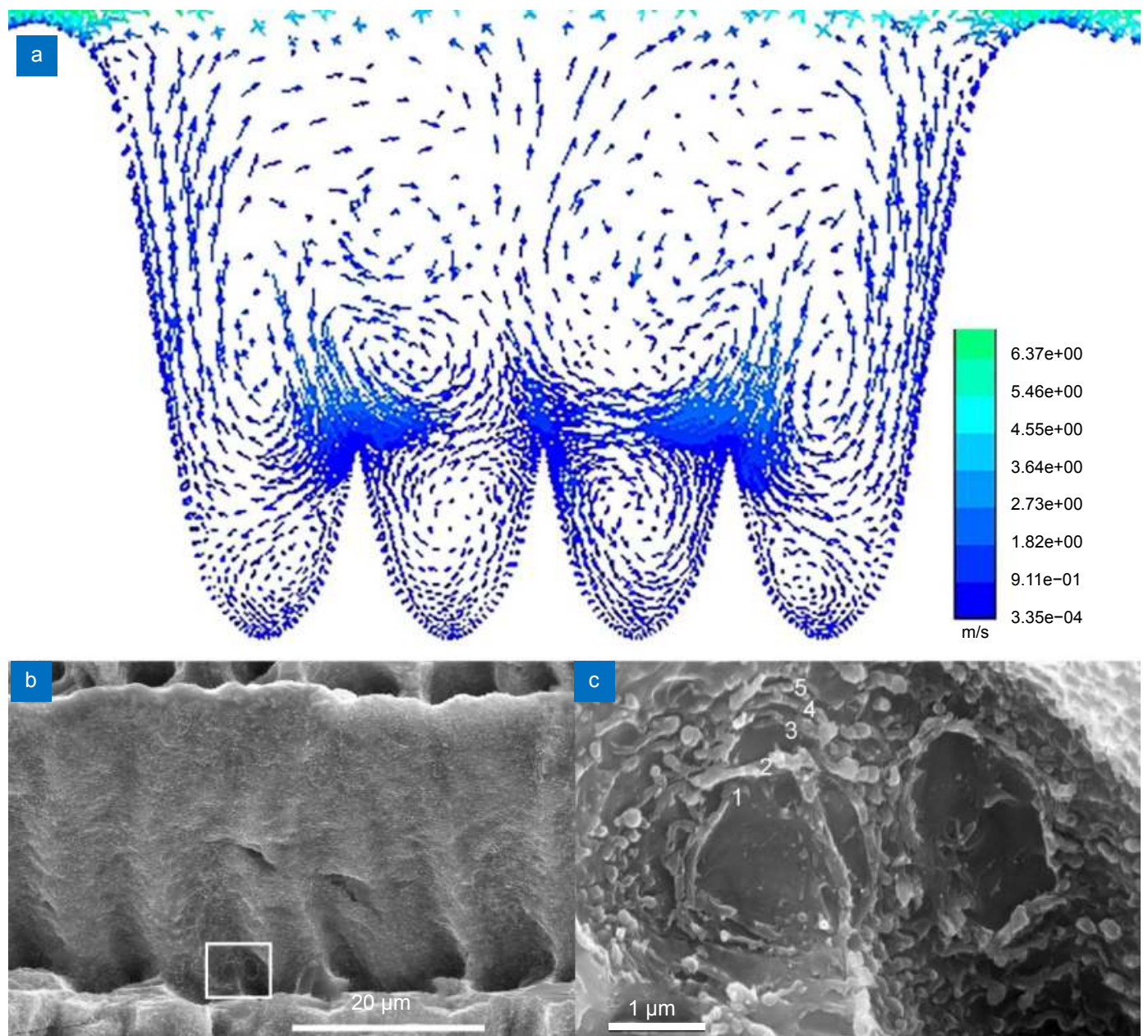

Fig. 3 | (a) Fluidic velocity vector in four consecutive macropores upon the impact of high speed liquids from both horizontal and vertical directions, which indicates the formation of liquid vortexes on the side walls of the groove, near the openings of the macropores. (b-c) SEM images of CLIPSS formed on the openings of the macropores which are produced by fs-LAL of $\mathrm{Si}$ in water under the conditions of a laser power of 700 $\mathrm{mW}$, a scan interval of $15 \mu \mathrm{m}$, and a scan speed of $1 \mathrm{~mm} / \mathrm{s}$. (c) Enlarged morphology of the white rectangle region in (b). 


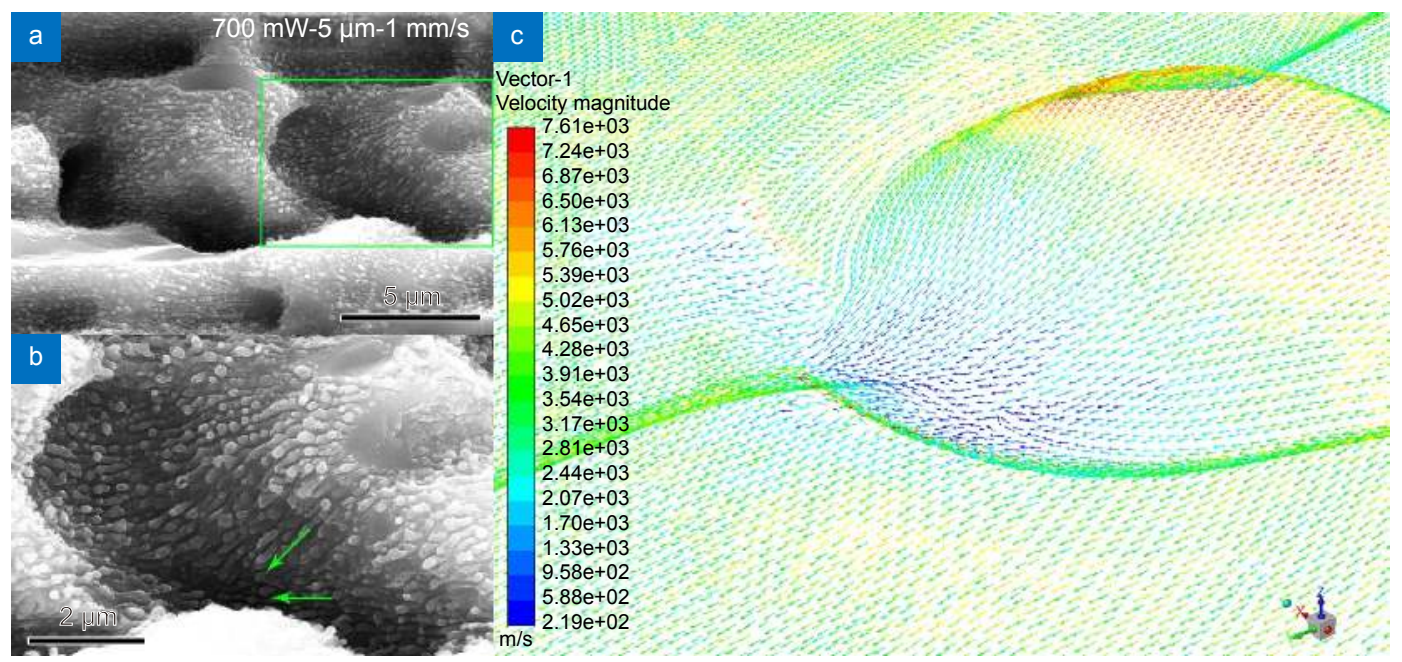

Fig. 4 | (a) SEM images of CLIPSS formed on the openings of macropores which are produced by fs-LAL of Si in water under the conditions of a laser power of $700 \mathrm{~mW}$, a scan interval of $5 \mu \mathrm{m}$, and a scan speed of $1 \mathrm{~mm} / \mathrm{s}$. (b) Enlarged morphology of the green rectangle in (a) which indicates the convergence of LIPSS oriented in two different directions. (c) Fluidic velocity vector of the flows while encountering a mountain-like structure like (b), which vary their directions at different heights of the structure. Simulation conditions: an enlarged mountain-like structure with height and radius of 6 and $10 \mu \mathrm{m}$, respectively. A small trench with a depth of $2 \mu \mathrm{m}$ is located beneath the mountain-like structure. The simulation model is a little enlarged compared with the structure shown in (b), which is aimed to ensure different directional flows on different parts of the structures to be seen more clearly. The starting flow is horizontal from top right to bottom left at a speed speed of $5 \mathrm{~km} / \mathrm{s}$.

simulates the fluid field on a mountain-like structure, which shows that the fluid direction alters when it encounters the surface structures with height gradients. The fluid can either climb the top of a mountain-like structure, surround the hillside or along the trench at the bottom of the mountain-like structure. Such a scheme makes the surface structures evolve into the mountainlike and simultaneously promises the recarving LIPSS on the crack surfaces (Fig. 4(b)) $)^{30}$.

Previously, CLIPSS has never been achieved by laser ablation using linearly polarized lasers without any help of external stimuli, but only achievable using an optical vortex beam ${ }^{82,83}$ or modulated beams ${ }^{42,84}$, or with the assistance of bubble diffraction ${ }^{43}$. The finding of both CLIPSS (Fig. 1(c-f)) and irregular HSFL (Fig. 1(i-k)) at the bottom and other positions of microholes (Fig. 3(b, c)) indicates that many vortex forces are generated during fs-LAL due to the ultrafast motions of strong, complex (vertical and horizontal) fluids. Conventionally, temperature-gradient-induced Marangoni flow and plasma-pressure-driven flow are considered to be the driving forces to stimulate the motion of molten layers from center to periphery of ablated craters ${ }^{85,86}$. In this work, we demonstrated that liquid flow including liquid vortexes produced by shockwaves or the collapse of cavitation bubbles ${ }^{3}$ during fs-LAL is another factor that can induce the movement of molten layers. During the movement of molten layers, they are engraved into LIPSS with the aid of surface plasmon polaritons. In the case of fs-LAL of brittle materials such as $\mathrm{W}$, the ultrahigh-pressure of liquid vortexes which exert to the side walls can cause severe horizontal cracks. At the higher laser power of $700 \mathrm{~mW}$ (pulse energy of $7 \mu \mathrm{J}$ ), both CLIPSS (Fig. 1(c-f), Fig. 3(c)) and CCLIPSS (Fig. $4(\mathrm{~b}))$ can be generated everywhere of the microstructures such as at the bottom and on the side walls of macropores and on the crests of broken grooves. In addition, mobile vortexes cause the formation of trenches on side walls of ablated grooves (Fig. 2). But at the lower laser power of $50 \mathrm{~mW}$ (pulse energy of $0.5 \mu \mathrm{J}$ ), only curved HSFL can be generated in the troughs and the crests of grooves (Fig. 1(j, k)). Figure 1(i, j) demonstrate the reproducibility of the formation of CLIPSS, while the reproducibility of CCLIPSSs can refer to our previous work ${ }^{48}$.

In our experiments, we adopted a fs laser with the repetition rate of $100 \mathrm{kHz}$, within the scope of multipulse experiments. We believe that our simulation results are effective for multipulse laser ablation for the following reasons. Shockwaves which are originated from laser ablation and bubble collapse are the driving force for the formation of high-speed fluids. Under the conditions of multipulse ablation, the directions of shockwaves should be similar to our simulated cases including both 
high-speed superimposed horizontal and vertical flows or horizontal flows and the velocity of fluids originated from pushing force of shockwaves should also be in the same order.

\section{Conclusions}

In conclusion, this paper presented the possibility to break through the optical limitation of linear polarization for formation of CLIPSS and CCLIPSS by the liquid vortexes and multi-directional liquid flows triggered during fs-LAL in water. The strength of liquid vortexes depends on the laser fluence. Higher laser fluence generates stronger liquid vortexes, which can lead to the selfformation of macroporous structures in the ablated grooves. In the case of fs-LAL of brittle metals such as W, the ultrahigh-pressure of liquid vortexes can cause severe cracks on the side walls of ablated grooves. On the mountain-like microstructures, fluids flow with altering directions, which can cause the formation of CCLIPSS. This finding can well explain the orientation deviation of Si-HSFL on the micro/nanostructures with height gradients we have reported previously ${ }^{48}$. Our findings indicate that LIPSS is a good indicator for restituting the fluid states during fs-LAL, which in turn highlights the importance of liquid dynamics for micro/nanostructuring during fs-LAL, therefore may inspire more attempts to be dedicated to tuning the structures of LIPSS via liquid maniputlation.

\section{References}

1. Phillips KC, Gandhi HH, Mazur E, Sundaram SK. Ultrafast laser processing of materials: a review. Adv Opt Photonics 7, 684-712 (2015).

2. Li L, Hong MH, Schmidt M, Zhong ML, Malshe A et al. Laser nano-manufacturing-state of the art and challenges. CIRP Ann 60, 735-755 (2011).

3. Zhang DS, Gökce B, Barcikowski S. Laser synthesis and processing of colloids: fundamentals and applications. Chem Rev 117, 3990-4103 (2017).

4. Sugioka K, Cheng Y. Ultrafast lasers -reliable tools for advanced materials processing. Light Sci App/ 3, e149 (2014).

5. Zhou R, Zhang Z, Hong MH. The art of laser ablation in aeroengine: the crown jewel of modern industry. J Appl Phys 127, 080902 (2020).

6. Grigoropoulos CP. Laser synthesis and functionalization of nanostructures. Int J Extrem Manuf 1, 012002 (2019).

7. Palneedi H, Park JH, Maurya D, Peddigari M, Hwang GT et al. Laser irradiation of metal oxide films and nanostructures: applications and advances. Adv Mater 30, 1705148 (2018).

8. Chen L, Cao KQ, Li YL, Liu JK, Zhang SA et al. Large-area straight, regular periodic surface structures produced on fused silica by the interference of two femtosecond laser beams through cylindrical lens. Opto-Electron Adv 4, 200036 (2021).

9. Zhang D, Liu R, Li Z. Irregular LIPSS produced on metals by single linearly polarized femtosecond laser. Int J Extrem Manuf 4, 015102 (2022).

10. Zhang D, Ranjan B, Tanaka T, Sugioka K. Carbonized Hybrid Micro/Nanostructured Metasurfaces Produced by Femtosecond Laser Ablation in Organic Solvents for Biomimetic Antireflective Surfaces. ACS Appl Nano Mater 3, 1855-1871 (2020).

11. Vorobyev AY, Guo CL. Direct femtosecond laser surface nano/microstructuring and its applications. Laser Photon Rev 7, 385-407 (2013).

12. Malinauskas M, Žukauskas A, Hasegawa S, Hayasaki $Y$, Mizeikis $\mathrm{V}$ et al. Ultrafast laser processing of materials: from science to industry. Light Sci App/ 5, e16133 (2016).

13. Liu R, Zhang DS, Li Z G. Femtosecond laser induced simultaneous functional nanomaterial synthesis, in situ deposition and hierarchical LIPSS nanostructuring for tunable antireflectance and iridescence applications. J Mater Sci Technol 89, 179-185 (2021).

14. Livakas N, Skoulas E, Stratakis E. Omnidirectional iridescence via cylindrically-polarized femtosecond laser processing. OptoElectron Adv 3, 190035 (2020).

15. Zhang CY, Zhou W, Geng D, Bai C, Li WD et al. Laser direct writing and characterizations of flexible piezoresistive sensors with microstructures. Opto-Electron Adv 4, 200061 (2021).

16. Stratakis E, Bonse J, Heitz J, Siegel J, Tsibidis GD et al. Laser engineering of biomimetic surfaces. Mater Sci Eng R Rep 141, 100562 (2020).

17. Shukla P, Waugh DG, Lawrence J, Vilar R. Laser surface structuring of ceramics, metals and polymers for biomedical applications: a review. Vilar R, ed. Laser Surface Modification of Biomaterials. 281-299 (Elsevier, 2016).

18. Zhang DS, Ranjan B, Tanaka T, Sugioka K. Underwater persistent bubble-assisted femtosecond laser ablation for hierarchical micro/nanostructuring. Int J Extrem Manuf 2, 015001 (2020).

19. Zhao LL, Liu Z, Chen D, Liu F, Yang Z et al. Laser synthesis and microfabrication of micro/nanostructured materials toward energy conversion and storage. Nano-Micro Lett 13, 49 (2021).

20. Wu ZP, Yin K, Wu JR, Zhu Z, Duan JA et al. Recent advances in femtosecond laser-structured Janus membranes with asymmetric surface wettability. Nanoscale 13, 2209-2226 (2021).

21. Wang XD, Yu HB, Li PW, Zhang YZ, Wen YD et al. Femtosecond laser-based processing methods and their applications in optical device manufacturing: a review. Opt Laser Technol 135, 106687 (2021).

22. Han JD, Zhang F, van Meerbeek B, Vleugels J, Braem A et al. Laser surface texturing of zirconia-based ceramics for dental applications: a review. Mater Sci Eng C 123, 112034 (2021).

23. Zhang B, Wang L, Chen F. Recent advances in femtosecond laser processing of $\mathrm{LiNbO}_{3}$ crystals for photonic applications. Laser Photon Rev 14, 1900407 (2020).

24. Zhang YY, Jiao YL, Li CZ, Chen C, Li JW et al. Bioinspired micro/nanostructured surfaces prepared by femtosecond laser direct writing for multi-functional applications. Int $J$ Extrem Manuf 2, 032002 (2020).

25. Jia YC, Wang SX, Chen F. Femtosecond laser direct writing of flexibly configured waveguide geometries in optical crystals: fabrica-tion and application. Opto-Electron Adv 3, 190042 (2020).

26. Wan ZF, Chen X, Gu M. Laser scribed graphene for supercapacitors. Opto-Electron Adv 4, 200079 (2021). 
27. Wang HT, Hao CL, Lin H, Wang YT, Lan T et al. Generation of super-resolved optical needle and multifocal array using graphene oxide metalenses. Opto-Electron Adv 4, 200031 (2021).

28. Maksimovic J, Ng SH, Katkus T, Cowie BCC, Juodkazis S. External field-controlled ablation: magnetic field. Nanomaterials $\mathbf{9}$, 1662 (2019).

29. Maksimovic J, Ng SH, Katkus T, Le NHA, Chon JWM et al. Ablation in externally applied electric and magnetic fields. Nanomaterials 10, 182 (2020).

30. Zhang DS, Wu LC, Ueki M, Ito Y, Sugioka K. Femtosecond laser shockwave peening ablation in liquids for hierarchical micro/nanostructuring of brittle silicon and its biological application. Int J Extrem Manuf 2, 045001 (2020).

31. Dittrich S, Barcikowski S, Gökce B. Plasma and nanoparticle shielding during pulsed laser ablation in liquids cause ablation efficiency decrease. Opto-Electron Adv 4, 200072 (2021).

32. Bonse J, Höhm S, Kirner SV, Rosenfeld A, Krüger J. Laser-induced periodic surface structures-a scientific evergreen. IEEE J Sel Top Quantum Electron 23, 9000615 (2017).

33. Buividas R, Mikutis M, Juodkazis $S$. Surface and bulk structuring of materials by ripples with long and short laser pulses: recent advances. Prog Quantum Electron 38, 119-156 (2014).

34. Birnbaum M. Semiconductor surface damage produced by ruby lasers. J Appl Phys 36, 3688-3689 (1965).

35. Tan DZ, Sharafudeen KN, Yue YZ, Qiu JR. Femtosecond laser induced phenomena in transparent solid materials: fundamentals and applications. Prog Mater Sci 76, 154-228 (2016).

36. Cerkauskaite A, Drevinskas R, Solodar A, Abdulhalim I, Kazansky PG. Form-birefringence in ITO thin films engineered by ultrafast laser nanostructuring. ACS Photonics 4, 2944-2951 (2017).

37. Lam B, Zhang JH, Guo CL. Generation of continuously rotating polarization by combining cross-polarizations and its application in surface structuring. Opt Lett 42, 2870-2873 (2017).

38. Han WN, Jiang L, Li XW, Liu PJ, Xu L et al. Continuous modulations of femtosecond laser-induced periodic surface structures and scanned line-widths on silicon by polarization changes. Opt Express 21, 15505-15513 (2013).

39. Reinhardt H, Kim HC, Pietzonka C, Kruempelmann J, Harbrecht $B$ et al. Self-organization of multifunctional surfaces - the fingerprints of light on a complex system. Adv Mater 25, 3313-3318 (2013).

40. Dusser B, Sagan Z, Soder H, Faure N, Colombier JP et al. Controlled nanostructrures formation by ultra fast laser pulses for color marking. Opt Express 18, 2913-2924 (2010).

41. Varlamova O, Costache F, Reif J, Bestehorn M. Self-organized pattern formation upon femtosecond laser ablation by circularly polarized light. App/ Surf Sci 252, 4702-4706 (2006).

42. Han W, Liu F, Yuan Y, Li X, Wang Q et al. Femtosecond laser induced concentric semi-circular periodic surface structures on silicon based on the quasi-plasmonic annular nanostructure. Nanotechnology 29, 305301 (2018).

43. Romashevskiy SA, Ashitkov SI, Agranat MB. Circular ripple patterns on silicon induced by bubble-diffracted femtosecond laser pulses in liquid. Opt Lett 45, 1005-1008 (2020).

44. Ouyang J, Perrie W, Allegre OJ, Heil T, Jin Y et al. Tailored optical vector fields for ultrashort-pulse laser induced complex surface plasmon structuring. Opt Express 23, 12562-12572 (2015).

45. Cheng HC, Li P, Liu S, Chen P, Han L et al. Vortex-controlled morphology conversion of microstructures on silicon induced by femtosecond vector vortex beams. Appl Phys Lett 111, 141901 (2017).

46. Nivas JJ, He ST, Song ZM, Rubano A, Vecchione A et al. Femtosecond laser surface structuring of silicon with Gaussian and optical vortex beams. App/ Surf Sci 418, 565-571 (2017).

47. Tsibidis GD, Skoulas E, Stratakis E. Ripple formation on nickel irradiated with radially polarized femtosecond beams. Opt Lett 40, 5172-5175 (2015).

48. Zhang DS, Sugioka K. Hierarchical microstructures with high spatial frequency laser induced periodic surface structures possessing different orientations created by femtosecond laser ablation of silicon in liquids. Opto-Electron Adv 2, 190002 (2019).

49. Tsibidis GD, Fotakis C, Stratakis E. From ripples to spikes: a hydrodynamical mechanism to interpret femtosecond laser-induced self-assembled structures. Phys Rev B 92, 041405 (2015).

50. Zhang DS, Chen F, Yang Q, Yong JL, Bian $\mathrm{H}$ et al. A simple way to achieve pattern-dependent tunable adhesion in superhydrophobic surfaces by a femtosecond laser. ACS Appl Mater Interfaces 4, 4905-4912 (2012).

51. Zhang DS, Ranjan B, Tanaka T, Sugioka K. Multiscale hierarchical micro/nanostructures created by femtosecond laser ablation in liquids for polarization-dependent broadband antireflection. Nanomaterials 10, 1573 (2020).

52. Nguyen TTP, Tanabe-Yamagishi R, Ito $Y$. Impact of liquid layer thickness on the dynamics of nano- to sub-microsecond phenomena of nanosecond pulsed laser ablation in liquid. App/ Surf Sci 470, 250-258 (2019).

53. Letzel A, Santoro M, Frohleiks J, Ziefuß AR, Reich S et al. How the re-irradiation of a single ablation spot affects cavitation bubble dynamics and nanoparticles properties in laser ablation in liquids. App/ Surf Sci 473, 828-837 (2018).

54. Zhang DS, Gökce B, Sommer S, Streubel R, Barcikowski S. Debris-free rear-side picosecond laser ablation of thin germanium wafers in water with ethanol. Appl Surf Sci 367, 222-230 (2016).

55. Florian C, Déziel JL, Kirner SV, Siegel J, Bonse J. The role of the laser-induced oxide layer in the formation of laser-induced periodic surface structures. Nanomaterials 10, 147 (2020).

56. Long JY, Eliceiri MH, Ouyang YX, Zhang YK, Xie XZ et al. Effects of immersion depth on the dynamics of cavitation bubbles generated during ns laser ablation of submerged targets. Opt Lasers Eng 137, 106334 (2021).

57. Zeng $X Z$, Mao XL, Mao SS, Wen SB, Greif R et al. Laser-induced shockwave propagation from ablation in a cavity. Appl Phys Lett 88, 061502 (2006).

58. Shen MY, Crouch CH, Carey JE, Younkin R, Mazur E et al. Formation of regular arrays of silicon microspikes by femtosecond laser irradiation through a mask. Appl Phys Lett 82, 1715-1717 (2003).

59. Shih CY, Streubel R, Heberle J, Letzel A, Shugaev MV et al. Two mechanisms of nanoparticle generation in picosecond laser ablation in liquids: the origin of the bimodal size distribution. Nanoscale 10, 6900-6910 (2018).

60. Sedao X, Shugaev MV, Wu CP, Douillard T, Esnouf C et al. Growth twinning and generation of high-frequency surface nanostructures in ultrafast laser-induced transient melting and resolidification. ACS Nano 10, 6995-7007 (2016).

61. Xu YZ, Zhu HR, Zheng J. Effect of Knudsen number on 
microscale similar flow characteristics. J Aerosp Power 28, 1752-1758 (2013).

62. Tanabe R, Nguyen TTP, Sugiura T, Ito Y. Bubble dynamics in metal nanoparticle formation by laser ablation in liquid studied through high-speed laser stroboscopic videography. Appl Surf Sci 351, 327-331 (2015).

63. Nakagawa A, Kumabe T, Ogawa Y, Hirano T, Kawaguchi T et al. Pulsed laser-induced liquid jet: evolution from shock/bubble interaction to neurosurgical application. Shock Waves 27, 1-14 (2017).

64. Gad-el-Hak M. The fluid mechanics of microdevices-the freeman scholar lecture. J Fluids Eng 121, 5-33 (1999).

65. Nguyen TTP, Tanabe R, Ito $\mathrm{Y}$. Laser-induced shock process in under-liquid regime studied by time-resolved photoelasticity imaging technique. Appl Phys Lett 102, 124103 (2013).

66. Babenko VA, Bunkin NF, Sychev AA. Role of gas nanobubbles in nonlinear hyper-Raman scattering of light in water. J Opt Soc Am B 37, 2805-2814 (2020).

67. Namura K, Imafuku S, Kumar S, Nakajima K, Sakakura M et al. Direction control of quasi-stokeslet induced by thermoplasmonic heating of a water vapor microbubble. Sci Rep 9, 4770 (2019).

68. Derrien TJY, Koter R, Krüger J, Höhm S, Rosenfeld A et al. Plasmonic formation mechanism of periodic 100-nm-structures upon femtosecond laser irradiation of silicon in water. $J \mathrm{App} /$ Phys 116, 074902 (2014).

69. Stratakis E, Zorba V, Barberoglou M, Fotakis C, Shafeev GA. Laser writing of nanostructures on bulk Al via its ablation in liquids. Nanotechnology 20, 105303 (2009).

70. Bonse J. Quo vadis LIPSS? - recent and future trends on laserinduced periodic surface structures. Nanomaterials 10, 1950 (2020).

71. Bonse J, Gräf S. Maxwell meets marangoni-a review of theories on laser-induced periodic surface structures. Laser Photonics Rev 14, 2000215 (2020).

72. Ji LF, Lv XZ, Wu Y, Lin ZY, Jiang YJ. Hydrophobic light-trapping structures fabricated on silicon surfaces by picosecond laser texturing and chemical etching. J Photonics Energy 5, 053094 (2015).

73. Zhang CY, Yao JW, Liu HY, Dai QF, Wu LJ et al. Colorizing silicon surface with regular nanohole arrays induced by femtosecond laser pulses. Opt Lett 37, 1106-1108 (2012).

74. Liu HG, Lin WX, Lin ZY, Ji LF, Hong MH. Self-organized periodic microholes array formation on aluminum surface via femtosecond laser ablation induced incubation effect. Adv Funct Mater 29, 1903576 (2019).

75. Yuan DQ, Wu QR, Xu JT, Zhou M, Yang HF. Periodic nanohole array structure induced on a silicon surface by direct writing with a femtosecond laser. J Opt Technol 82, 353-356 (2015).

76. Perry AE, Chong MS, Lim TT. The vortex-shedding process be- hind two-dimensional bluff bodies. J Fluid Mech 116, 77-90 (1982).

77. Akram M, Bashir S, Rafique MS, Hayat A, Mahmood K et al. Morphological and spectroscopic characterization of laser-ablated tungsten at various laser irradiances. Appl Phys A 119, 859-870 (2015).

78. Gu DD, Guo M, Zhang HM, Sun YX, Wang R et al. Effects of laser scanning strategies on selective laser melting of pure tungsten. Int J Extrem Manuf 2, 025001 (2020).

79. Tuerke F, Pastur LR, Sciamarella D, Lusseyran F, Artana G. Experimental study of double-cavity flow. Exp Fluids 58, 76 (2017).

80. Liu Y, Cui J, Jiang YX, Li WZ. A numerical study on heat transfer performance of microchannels with different surface microstructures. Appl Therm Eng 31, 921-931 (2011).

81. Wang W, Zhang YN, Li BX, Han HZ, Gao XY. Influence of geometrical parameters on turbulent flow and heat transfer characteristics in outward helically corrugated tubes. Energy Convers Manag 136, 294-306 (2017).

82. Nivas JJ, He ST, Rubano A, Vecchione A, Paparo D et al. Direct femtosecond laser surface structuring with optical vortex beams generated by a q-plate. Sci Rep 5, 17929 (2015).

83. Lou K, Qian SX, Wang XL, Li YN, Gu B et al. Two-dimensional microstructures induced by femtosecond vector light fields on silicon. Opt Express 20, 120-127 (2012).

84. Han WN, Li DF, Liu FR, Yuan YP, Li XW. Controllable formation of si nanostructures based on quasi-plasmonic planar nanostructures formed by annular-shaped femtosecond laser pulse. IEEE Photonics J 11, 2400208 (2019).

85. Ben-Yakar A, Byer RL, Harkin A, Ashmore J, Stone HA et al. Morphology of femtosecond-laser-ablated borosilicate glass surfaces. Appl Phys Lett 83, 3030-3032 (2003).

86. Ben-Yakar A, Harkin A, Ashmore J, Byer RL, Stone HA. Thermal and fluid processes of a thin melt zone during femtosecond laser ablation of glass: the formation of rims by single laser pulses. J Phys D Appl Phys 40, 1447-1459 (2007).

\section{Acknowledgements}

This work was supported by the national key R\&D program for international collaboration under grant No. 2018YFE9103900, and in part by the Natural Science Foundation of China (NSFC) grant No. 11972384 and the Guangdong MEPP Fund, grant No. GDOE [2019] A01.

\section{Author contributions}

D. S. Zhang and K. Sugioka proposed the original idea. D. S. Zhang fabricated the samples and performed the measurements. X. Z. Li, Y. Fu, Q. H. Yao performed the simulations. Q. H. Yao, Z. G. Li, and K. Sugioka revised the manuscripts.

\section{Competing interests}

The authors declare no competing financial interests. 